\title{
DEPORTE ENTRE REJAS ¿Algo más que control social?
}

\author{
SPORT BEHIND BARS \\ Anything beyond social control?
}

\author{
Daniel Martos García \\ Universitat de València. Estudi General'. España \\ daniel.martos@ucv.es
}

José Devís Devís

Universidad Católica de Valencia. España

jose.devis@uv.es

\author{
ANDREW C. SPARKES \\ University of Exeter. Reino Unido \\ a.c.sparkes@exeter.ac.uk
}

\section{Resumen}

Las actividades deportivas forman parte del paisaje de las prisiones. Sin embargo, escasea el conocimiento sobre el papel que desempeñan en la vida cotidiana de las cárceles. Por ello realizamos un estudio etnográfico, principalmente en el polideportivo de una prisión española, dirigido a comprender los significados que el ejercicio físico y el deporte tenían para los presos, funcionarios, educadores y monitores. Los resultados indican que la reinserción mediante estas prácticas es una ilusión, a pesar de ser la finalidad oficial de la reclusión. Aun así, les asignan diversos beneficios, especialmente los presos y presas. Entre ellos se encuentra el potencial educativo ligado al autocontrol y, sobre todo, el entretenimiento y la compensación para ocupar el tiempo, huir del hastío y sobrellevar los problemas del encierro. El artículo concluye que el ejercicio y el deporte pueden tener un papel de "evasión" simbólica y liberación personal, aunque los presos viven atenazados por un contexto en el que prima el orden y el control por encima de todo.

\section{Palabras Clave Adicionales}

Compensación, Ejercicio físico, Etnografía, Prisión, Reinserción.

\section{Abstract}

Sport practices belong to the prison's landscape. Nevertheless, there is a lack of knowledge about the role they play in prisons ordinary life. Therefore, we developed an ethnographic study, mainly located in the sports hall of a Spanish prison, to understand exercise and sport meanings among inmates, workers, educators and monitors. Data indicate that social rehabilitation through sport is an illusion, although it is the official goal of imprisonment. Even so, participants assign different benefits to exercise and sport, especially inmates. Educative potential of self-control through sport and, mainly, distraction and compensation strategy to cope with boredom, time and confinement's physical and mental problems are among them. Paper concludes that

${ }^{1}$ Agradecemos al Ministerio de Educación y Ciencia la ayuda de movilidad concedida a José Devís (Resolución de la Secretaría de Estado de Universidades e Investigación de 15 de abril de 2005) porque ha permitido coordinar la redacción del artículo. 
exercise and sport can play a symbolic 'evasion' and personal liberation, but are constrained by a context in which control and order are at the forefront of everything.

Additional KeYwords

Compensation, Exercise, Ethnography, Prison, Rehabilitation.

\section{INTRODUCCIÓN}

En la actualidad, las instalaciones y prácticas físicas y deportivas forman parte del paisaje de las prisiones de los países occidentales. En ello contribuyó, según Hagan (1989), el rol desempeñado por el deporte y la recreación en el denominado 'Experimento de Bristol', un estudio realizado a finales de la década de 1960 en Reino Unido, en el que se señalaban algunos beneficios para la reinserción vinculados a la estrategia de ofrecer cierta libertad de asociación a los presos. Para este autor, la introducción de las actividades deportivas es una consecuencia más del cambio de papel asignado a las prisiones cuando pasaron de ser centros de castigo y revancha a centros orientados a la rehabilitación social. De acuerdo con Foucault (1978: 16), a partir del siglo XVIII y principios del XIX las cárceles abandonan progresivamente "la sombría fiesta punitiva" y se sumergen en las ideas de reintegración social. A las personas presas, a pesar del encierro al que estaban sometidas, se les considera seres humanos con derechos y, por esta razón, las actividades recreativas al aire libre van incorporándose como una parte más de las necesidades de la vida diaria de una prisión. En definitiva, la reinserción se convierte en la nueva ideología oficial del sistema penitenciario de las sociedades democráticas y reserva un lugar al deporte en la vida carcelaria (Caplan, 1996).

El Estado español no es, en este sentido, ninguna excepción. Así lo indica la Constitución en su artículo 25.2, donde se manifiesta que las prisiones están pensadas y construidas con la intención de reeducar y reinsertar en la sociedad a las personas condenadas (Constitución Española, 1983). Además, en la Ley Orgánica General Penitenciaria (LOGP, 1979), derivada de la Carta Magna, se mencionan las instalaciones deportivas y la posibilidad de organizar y participar en actividades de esta índole. Asimismo, el Reglamento Penitenciario (1997) expresa, en el capítulo denominado Formación, cultura y deporte, que las actividades educativas, formativas y deportivas están determinadas por el Consejo de Dirección del centro según propuesta de la Junta de Tratamiento. En su artículo 131, el texto señala que se realizarán las actividades deportivas más convenientes para conseguir el desarrollo integral de las personas encarceladas. Es decir, que el deporte en los textos penitenciarios encuentra lugar en el apartado reservado a las actividades encaminadas a la reinserción social, dentro del conjunto de artículos dedicados a la educación.

Pero más allá de los textos legales, nos llama la atención el limitado conocimiento sobre el ejercicio físico y el deporte dentro de la vida carcelaria. Hay instituciones que manifiestan la conveniencia de la realización de prácticas físicas en la prisión, como la Asociación Americana de Corrección que recomienda a los presos y presas la 
realización de, al menos, una hora de ejercicio al día (Hitchcock, 1990). De igual forma, encontramos varios autores que han investigado o escrito sobre la relación positiva entre ejercicio y salud dentro de las prisiones (Courtenay y Sabo, 2001; Hagan, 1989; Mortimer, 1999; Wagner, MaBride y Crouse, 1999). En el contexto español, también existen autores entusiastas del potencial saludable y rehabilitador de la práctica física realizada por parte de presos y presas (Castillo, 2005 y 2006; Chamarro, 1993; Chamarro, Blasco y Palenzuela, 1998). Otros argumentan que el ejercicio mejora la calidad de vida (Negro, 1995) y contribuye a la ocupación del tiempo libre, la evasión y la liberación de la agresividad (Ríos, 2004).

Sin embargo, escasean las aportaciones etnográficas sobre el papel que desempeña el ejercicio y el deporte en la vida cotidiana de las personas que viven y trabajan en las prisiones. Como señala Rhodes (2001), los obstáculos y las dificultades para hacer investigación en las prisiones no deberían frenar a los investigadores sociales si de verdad quieren comprender los significados presentes en estas instituciones y desafiar al lenguaje, los símbolos y las prácticas sociales que mantienen el sufrimiento y el castigo dentro del sistema penitenciario. En este sentido, el deporte y el ejercicio, como cualquier otra práctica social, son símbolos y medios comunicativos llenos de significado con los que las personas comparten sus experiencias y expectativas, se sitúan en el seno de una comunidad y sirven para construir su identidad. En uno de los pocos estudios etnográficos, realizado en los Estados Unidos de América, Grayzel (1978) mencionaba que el aburrimiento puede convertirse en el peor castigo para los presos y en una fuente de problemas para la convivencia dentro de la institución. Por esta razón señalaba que, en última instancia, las actividades estaban al servicio del control social. Más recientemente, Sabo (2001) muestra cómo el deporte y el ejercicio ayudan a los internos a pasar el tiempo en una prisión norteamericana de hombres. Además, este autor menciona que el deporte puede ser, simultáneamente, una fuente de liberación personal y una herramienta para el control social, dependiendo en gran medida de los significados que cada uno le otorgue a su práctica.

Como el ejercicio físico y el deporte cambian en el espacio y el tiempo, también cambian sus significados desde el momento en que son objeto de negociación dentro de un contexto. La interacción entre la gente y su pensamiento están íntimamente relacionados con su contexto y sus propósitos (Mejía, 2002). En el caso de la prisión, las actividades están influenciadas por los significados y las interpretaciones que las personas llevan del exterior, así como las creadas por la interacción constante dentro de la subcultura particular de cada centro penitenciario. Por lo tanto, la intersubjetividad y los símbolos compartidos también permitirán la emergencia de significados diferentes (Maykut y Morehouse, 2000). Por este motivo realizamos un estudio etnográfico dirigido a comprender los significados que el ejercicio físico y el deporte tenían para los implicados, directa e indirectamente, en las prácticas realizadas en el polideportivo de una prisión española de nuestros días (presos, funcionarios, educadores, voluntarios y otros cargos). En este artículo, reflejamos la parte del estudio que gira alrededor del control social, la compensación y la reinserción. 


\section{Metodología}

La estrategia metodológica utilizada es la etnografía, mediante la cual se describe e interpreta la vida de un grupo humano durante un período de tiempo prolongado. En este caso se trata de una etnografía realizada, durante algo más de dos años (2001-2003), en la sección de penados de una prisión española de alta seguridad y, especialmente, alrededor de las prácticas físicas realizadas en el polideportivo.

El trabajo de campo lo llevó a cabo el investigador principal (Daniel Martos) con la negociación formal (oficial) e informal del acceso a la prisión, tras convencer a las personas clave del interés de la investigación. Los permisos y el consentimiento oficial los consiguió a través de su participación en una ONG. para colaborar con el educador de deportes de la prisión. Eso incluía una tarea de ayuda profesional como voluntario, además de la tarea investigadora. Detrás quedaban muchos meses de espera y la evidencia de la dificultad que entraña entrar oficialmente en la prisión. El largo proceso reafirma la tradicional reticencia de las instituciones penitenciarias a abrir sus puertas a la investigación (Cabrera y Ríos, 1998). Aun así, tener acceso al recinto no garantiza la inmersión en la cultura carcelaria y la obtención de datos de calidad. Debía conseguir, además, la confianza de los y las participantes. Como dice Woods (1998) el éxito final depende mucho de la capacidad de una persona para venderse como digna y con un proyecto de valor. En este punto fue decisivo el educador de deportes, con quien iba a colaborar Daniel Martos, ya que actuó como portero de entrada al polideportivo y modo de acceso informal a los presos. Pero el rapport se consiguió gracias al rol de voluntario que ocupaba el investigador principal dentro del recinto penitenciario y su capacidad por mostrarse comprensivo y sensible a las preocupaciones de dentro. La tarjeta naranja que identificaba a los voluntarios le alejaba de cualquier connivencia institucional a los ojos de los presos y de cualquier rol de control externo para los funcionarios.

La forma que adoptó el acceso se acercaba a lo que algunos han denominado comercio justo en el contexto de la negociación entre profesores e investigadores (Goodson y Fliesser, 1995). La negociación del acceso resultaba beneficiosa para las principales partes implicadas, es decir, la ONG, la prisión, el educador de deportes, los presos y los investigadores. La ONG conseguía introducir un proyecto oficial en la prisión, el centro penitenciario aumentaba su plantilla en el polideportivo sin mermar sus precarios presupuestos, el educador conseguía una ayuda extra a su colapsada labor diaria, los presos y presas veían cómo se ponía en marcha una actividad al aire libre que les permitiría salir al exterior y los investigadores veían abiertas definitivamente las puertas de la prisión a sus necesidades de investigación.

Una vez dentro de la prisión, la información se recogió mediante los siguientes tres instrumentos:

- La observación, combinada con la participación, se llevó a cabo según el momento y la situación. En ocasiones únicamente se observaba lo que pasaba en el polideportivo, mientras que en otros momentos se llegaba a participar plenamente en las actividades. La observación se centraba principalmente en las personas y sucesos que ocurrían 
en el recinto del polideportivo, es decir, los presos participantes en las actividades y las personas encargadas de su desarrollo. También se visitaron otras dependencias del establecimiento (módulos y áreas comunes, fundamentalmente) y el investigador principal convivió con presos y presas en actividades organizadas fuera del recinto. De acuerdo con las necesidades de cada momento, se osciló de una observación total a una participación total, siguiendo los esquemas descritos por Wolcott (1973). Durante la duración de la investigación, la observación evolucionó de las primeras etapas de permanencia prolongada e intensa a otras finales de visitas puntuales combinadas con entrevistas (ver tabla 1). Las notas procedentes de las quinientas horas de observación las recogió el investigador en su diario de campo.

- La entrevista fue otro instrumento utilizado a lo largo de la investigación. En concreto, se realizaron cuarenta y seis entrevistas semidirigidas, de 40 minutos a una hora de duración, a menudo acompañadas de notas procedentes de las conversaciones informales que les precedieron y siguieron. El investigador estableció unas preguntas básicas sobre las que giraba la conversación, conforme a un guión general previo que variaba según el desarrollo de la entrevista y la persona entrevistada. Se realizaron a treinta y nueve personas, entre presos, funcionarios y voluntarios de la prisión de ambos géneros (27 hombres y 12 mujeres), la mayoría dentro del recinto (con y sin grabadora). Las transcripciones de las mismas las efectuó el investigador principal y posteriormente las entregaba en papel a la persona entrevistada para obtener la aprobación definitiva.

- Los documentos recopilados fueron de diversa índole. Entre ellos, la LOGP y otras normativas penitenciarias vigentes, así como ciertos documentos internos de la prisión que recogimos para ampliar la información y, en otros casos, contrastar los datos.

Tabla 1.

La observación y las entrevistas durante el trabajo de campo.

\begin{tabular}{lll}
\hline TIEMPO & OBSERVACIONES & ENTREVISTAS \\
\hline febrero-junio 2001 & $\begin{array}{l}\text { - Observación intensa: 4-5 días semanales } \\
\text { (9h. a 13h.) }\end{array}$ & $\begin{array}{l}\text { - Educador deportes: } \\
\text { 4 entrevistas }\end{array}$ \\
julio-agosto 2001 & - Descanso (visitas aisladas) & - Sin entrevistas \\
septiembre 2001- & - Observación constante: & - Presos: 20 entrevistas. \\
junio 2002 & 3 días semanales. & - Trabajadores y voluntarios: 8 \\
julio-agosto 2002 & - Descanso & - Sin entrevistas \\
septiembre- & - Observación constante: & - Presos: 1 entrevista \\
diciembre 2002 & 2 días semanales & - Trabajadores y voluntarios: 5 \\
enero-junio 2003 & - Observación puntual: 1 día a la semana & - Presos: 2 entrevistas \\
\hline
\end{tabular}

Hay que resaltar, en este punto, que el verdadero instrumento de recogida de datos en una etnografía es el investigador, por lo que las fuentes de recogida de información 
utilizadas en este estudio son simples recursos en manos del investigador principal. Los datos clave para captar la vida en el polideportivo y en la prisión son las palabras de los participantes y las notas de campo procedentes de las observaciones referidas a las descripciones, las emociones, las expectativas, las percepciones y los comportamientos. Estos datos se comenzaron a analizar al poco tiempo de iniciar la redacción de las notas de campo en el diario de observaciones del investigador principal, ya que demandaban ser ordenadas y dotadas de sentido. En estos primeros pasos de la investigación, Hammersley y Atkinson (1994) proponen leer los datos en pro de su familiarización, con el fin de extraer cuestiones significativas e identificar aspectos confusos o modelos que nos puedan interesar. Aun siendo un proceso flexible, y muchas veces fruto de la intuición, el análisis no dejó de ser consciente y dirigido y, sin duda, una etapa muy reflexiva. Tal y como señalan Taylor y Bogdan (1986), fue una especie de descubrimiento en proceso en el que extraíamos conceptos, cuestiones significativas, palabras clave pronunciadas por los propios participantes o bien identificamos frases y párrafos que hablaban del mismo asunto, susceptibles, pues, de ser agrupadas en la misma categoría. El objetivo no fue otro que el de reducir los datos para proporcionarles sentido y permitir la redacción de los oportunos informes y relatos. En definitiva, se trataba de permitir la extracción de significados e interpretaciones.

El proceso se complicó con el análisis de las entrevistas y documentos, ya que se sucedieron las rectificaciones para refinar las interpretaciones, fruto de la necesidad de adaptar las categorías a los nuevos datos. La cantidad de categorías no es un asunto baladí, por lo que se recomienda prestar mucha atención a este asunto. Por una parte, unas pocas categorías favorecen el manejo de los datos pero dificultan la localización de sucesos, mientras que, por otra parte, muchas categorías propician una alta ordenación de los datos pero muy descontextualizados (Coffrey y Atkinson, 1996). En nuestro estudio, inicialmente el listado de categorías fue muy largo por lo que se reestructuró para simplificarlo hasta que, definitivamente, quedó con un número de categorías y subcategorías que permitían reconstruir, según nuestro juicio, la vida en la prisión y los significados que otorgaban las personas implicadas en la práctica de actividades físicas y deportivas.

Una vez completado el análisis, abordamos la escritura del informe cualitativo. No es nada fácil plasmar en el papel aquello que queremos, decir, no es nada fácil, ahora bien, esta tarea puede verse facilitada si contamos con datos suficientes y un análisis conveniente que permita la emergencia de frases con sentido. En cualquier caso hay que señalar que la escritura es una prolongación del análisis, porque escribir es, en definitiva, reflexionar (Wolcott, 1990; Woods, 1998).

Finalmente, debemos mencionar que el valor de la verdad y el control de la confianza de los datos que dan rigor a nuestro estudio estaban apoyados, además de por el rapport mencionado más arriba, por:

- Un comportamiento ético. Aparte de cumplir los compromisos adoptados con las personas clave de la prisión, el investigador principal respetó lo que prometía en relación con las entrevistas y las observaciones. Básicamente, protección de las fuentes, no delatar a nadie y no juzgar lo que veía o sabía. 
- Una presencia prolongada en el contexto de estudio para dotar de credibilidad a una investigación (Fetterman, 1998). Se trata de una presencia de dos años, si bien con variación de intensidad durante este tiempo, tal y como se ha comentado al referirnos a la observación.

- La comprobación con los participantes de los textos elaborados a lo largo del proceso de investigación (Fetterman, 1998). Los informantes recibieron las transcripciones de las entrevistas y también los informes y relatos en los que estaban implicados para obtener sus sugerencias, críticas y conformidad.

- La triangulación o cruce de información procedente de diferentes instrumentos, grupos de estudio, escenarios y perspectivas teóricas en relación a un fenómeno (Flick, 2004). En concreto, se triangularon instrumentos como la observación, la entrevista y los documentos; los escenarios como el polideportivo, otras dependencias y actividades exteriores a la prisión; así como la triangulación de personas pertenecientes a diferentes grupos de participantes como presos, educadores, funcionarios y voluntarios.

En nuestro estudio, estas estrategias son una especie de precauciones para que la narración final resulte creíble a los lectores y lectoras, pero es imposible que los datos fluyan libres de valoraciones. A pesar de ello, no podemos garantizar la veracidad en el sentido en que lo hacen las investigaciones positivistas que buscan la correspondencia de la narración con la realidad investigada. La veracidad descansa, en última instancia, en la coherencia entre las diferentes partes del informe, la evidencia presente en el relato y la lógica utilizada en la interpretación. La verdad, como señalan Bolívar, Domingo y Fernández (2001) y Sparkes (2002), es fundamentalmente narrativa porque descansa en la capacidad del texto para persuadir y convencer.

\section{EL CONTEXTO}

"Varoic" (pseudónimo) es una institución penitenciaria española que cuenta con una población carcelaria de aproximadamente 2500 personas. Está compuesta por módulos que se dividen en dos secciones, una dedicada a los presos preventivos y otra a los condenados. Esta última sección, en la que se realizó el estudio etnográfico, consta de 12 módulos para unos 1300 presos, que se distribuyen fundamentalmente por el tipo de condena. Las celdas son de uso doble para personas del mismo sexo, aunque inicialmente fueron concebidas para una sola persona. Las mujeres se encuentran en un módulo y en otro de carácter mixto que está en régimen de experimentación. Cada módulo cuenta con un patio que incluye una cancha de futbito y el conjunto de la cárcel cuenta con una enfermería y un polideportivo.

Los presos pueden realizar prácticas físicas en el patio del módulo o en el polideportivo. En el patio las pueden realizar por libre en las horas en que tienen regulado el uso de este espacio. En el polideportivo pueden acudir en horario libre por las tardes (de 16.45 a 19.30 horas) y los fines de semana (de 10.30 a 13 horas y de 
16.45 a 19.30 horas). Durante los días de la semana, las mañanas están reservadas para las actividades y programas que forman parte del Tratamiento, es decir, el área que aglutina las propuestas dirigidas a la reinserción y (re)educación de los presos. El otro gran pilar en el funcionamiento de la prisión es el Régimen disciplinario que se ocupa del orden y la seguridad para facilitar la convivencia que permita cumplir las finalidades de la institución (LOGP, 1979).

Las prácticas físicas y deportivas de carácter (re)educativo las controla la Subdirección de Tratamiento pero, en la práctica, el educador de deportes es quién decide todos los asuntos relacionados con el polideportivo y las actividades que allí se realizan. Es decir, Alex (pseudónimo), además de decidir el horario y las actividades, también es el responsable de elaborar la lista de internos participantes, elegir los monitores que de vez en cuando contrata la prisión, y de seleccionar a los presos destinados a tareas de limpieza y enseñanza en el polideportivo (los "destinos"). Alex y los monitores contratados sólo trabajan por las mañanas, así que los destinos son los encargados de controlar y desarrollar las actividades de las tardes y los fines de semana.

Las actividades matutinas del Tratamiento son un privilegio para quienes pueden realizarlas porque se dirigen a un grupo selecto de presos y las supervisa personal especializado. Incluye las artes marciales (aikido, full-contact y kickboxing), el culturismo, el fútbol sala, la preparación para las actividades al aire libre, el squash, el yoga, la natación en verano, y un curso profesional para monitores deportivos. Los participantes no superan el $10 \%$ de los internos, mientras que se observa un uso masivo del polideportivo por las tardes. En ello influye el carácter voluntario de la participación, así como la competencia con otras actividades culturales y talleres de introducción a determinados oficios, que forman parte del Tratamiento y no pueden compaginarse. A esto deben añadirse los criterios de selección que realiza Alex con la ayuda de monitores y algún destino mediante entrevistas y recomendaciones de otros presos. Los criterios habituales son la buena conducta, la puntualidad, la participación continua o unas buenas condiciones físicas. La experiencia deportiva anterior es importante pero no resulta definitiva. Por iniciativa del educador de deportes, existe un club de artes marciales que participa en competiciones fuera de la cárcel. Especial atención establece Alex a la selección de sus miembros, ya que a los criterios habituales añade los de no crear problemas, no ser agresivos, no tener delitos de sangre ni antecedentes por violación.

\section{El EJeRCiCIO Físico Y EL DEPORTE ENTRE REJAS: LA PERSPECTIVA DE LOS PARTICIPANTES}

\section{¿Deporte y reinserción?}

El ejercicio físico y el deporte forman parte, como el resto de actividades del Tratamiento, de las prácticas educativas que tienen como meta oficial la reinserción de los presos y presas, tal y como se desprende de los textos legales. Sin embargo, los actores de 
nuestro estudio etnográfico no ven clara la meta de la reinserción, aunque consideran que el ejercicio físico puede ayudar a los encarcelados. Una de las reclusas deportistas lo manifestaba así: "... sales mejor [de la prisión]. Pero cuenta, que aquí hay mucha gente que hace deporte y después sigue con el tema [de la delincuencia]". Otro preso consideraba que el deporte no elimina la actividad delictiva, pero puede aportar algunos beneficios:

El deporte no te quita las ganas de atracar. Una persona que atraca, por hacer deporte no va a dejarlo. Pero con la droga es diferente. Me veo más preparado, más centrado, más adulto, más curtido.

Algunos perciben que el deporte puede ayudar a la relación social e insisten en que les aleja del consumo de drogas, tal y como menciona esta monitora:

Podía intentarse para inculcar unos valores, disciplina, y de comunicación social y de relación entre ellos que está totalmente olvidados [...] Por lo que he visto allí, la actividad física seria lo único que podría contribuir a intentar cambiar a la persona dentro [...] Y de hecho, yo sé de gente que va haciendo deporte, sale a full-contact, sale a fútbol-sala, sale a baloncesto y eran drogadictos y el deporte les ha venido de maravilla.

Los más optimistas consideran que pueden aprender alguna actividad profesional dentro de la prisión relacionada con el deporte que puede servirles cuando estén fuera, tal y como señala este preso:

Bueno, también te puede ayudar de forma profesional a buscarte un trabajo. Puedes hacer un curso aquí, ahora se hace el de monitor y el otro día oí en la tele que se necesitan socorristas para el verano. Pues aquí se han hecho varios cursos de socorrista.

Pero este potencial para la reinserción que menciona la monitora y el interno no está asociado a evitar la reincidencia delictiva. En este sentido, Alex, el educador de deportes del centro penitenciario, ve la reinserción mediante el deporte como un objetivo desenfocado y poco realista:

No te voy a decir que mediante el deporte se consiga la rehabilitación y reinserción social. Desde luego aquí en prisiones sería un poco tonto considerar ese objetivo como primordial [...] yo me marco unos objetivos personales, es decir, objetivos claros que se puedan conseguir, no la rehabilitación y reinserción del interno.

La reinserción es un proceso complejo y resulta muy ambicioso otorgar este potencial a la actividad física y deportiva. Como decía un monitor, la reinserción "no sólo depende del deporte", "el deporte es sólo una cosa más" insistía un trabajador social. Además, cuando la reinserción incluye la no reincidencia, hay que entender y actuar sobre las causas que provocaron previamente el delito (Alarcón, 1987) y el deporte, así como la misma prisión, presentan evidentes limitaciones en este sentido. Una subdirectora de 
tratamiento penitenciario manifestaba que la reinserción no puede ser una meta de la prisión porque ésta se consigue fuera de los muros carcelarios:

Nuestros objetivos son más específicos, más limitados. Nuestro alcance es mucho más limitado, nosotros tratamos con las personas mientras cumplen la condena; después, ya no podemos hacer nada. Y la reinserción se produce fuera y allí ya no llegamos.

Podemos ver, pues, que la sociedad juega un papel decisivo en la reinserción, excediendo claramente la actividad deportiva y a todo el programa de tratamiento de una prisión, tal y como señala este monitor:

Cualquier actividad [...] te mantiene vivo, hace que estés vivo, activo y no seas un florero. Te crea inquietudes porque en la prisión hay muy pocas. Pero la reinserción no existe por culpa de la sociedad. En la calle a los presos los rechazan [...] Los presos necesitan ayuda, un trabajo y un lugar para vivir. Aquí toman drogas y necesitan ayuda. Las prisiones sólo castigan. Caes preso y cuando sales te debes apañar tú solo.

Esta tarea individual que exige la reinserción en las condiciones sociales de vida actual son, precisamente, las que señalan algunos presos cuando abordan la cuestión. Un preso lo expresa diciendo que "si hay reinserción es voluntaria, pero la prisión no reinserta". Son muchas las alusiones al esfuerzo personal como la única manera de reinsertarse, como si se tratase de una especie de lucha de la persona contra las condiciones sociales, tal y como apunta esta interna: "Se reinserta la persona. La cárcel no ayuda a que una persona se reinserte". De alguna manera, el papel que juega la sociedad y el individuo en la reinserción recuerdan la relación compleja entre la estructura social y la agencia individual. Es decir, la voluntaria acción de las personas dentro de los condicionantes históricos y sociales en que viven, en este caso, una vez se extinguen sus condenas.

\section{Entretenimiento, compensación (y supervivencia)}

Entre las líneas del apartado anterior se han deslizado diversas manifestaciones que apuntan hacia un papel ampliamente reconocido del ejercicio físico y el deporte por parte de los diferentes actores del estudio. Nos referimos a una especie de compensación o evasión de los problemas y consecuencias negativas del encierro. Los problemas físicos, psicosociales y de salud que provoca el aislamiento (ver Courtenay y Sabo, 2001; Manzanos, 1992; Valverde, 1991) hacen del deporte algo más que una experiencia excitante para convertirse en un medio para soportar el sufrimiento y el confinamiento de los internos. Como decía un preso, "lo hago [el deporte], principalmente porque me gusta, pero también por distraerme y liberarme de este sitio. Cuando haces deporte no piensas que estás en la cárcel". De manera semejante lo expresa esta interna: 
Si salgo al poli es porque me siento bien... no sé cómo explicarlo, me olvido que estoy presa, me relajo. Pero no es por tener buen tipo, lo que pasa es que me siento limpia y elimino estrés.

El papel del deporte como compensación del estrés acumulado por el encierro es uno de los aspectos más mencionados. Para el educador de deportes "a través del deporte todo el estrés, parte del estrés que se genera en prisión se logra evitar" y, de manera parecida, se manifestaba un maestro de la institución cuando decía que "el deporte es básico, elimina el estrés a través del ejercicio físico". Sin embargo, probablemente, quien mejor lo refleja es este preso que lo vive en propia carne:

Mira, en la cárcel uno de los principales problemas, si no el que más, es el estrés. El estrés no te deja estudiar, no te deja relacionarte y eso se quita con el deporte [...] Es que aquí hay dos caminos o droga o lo que tú te montes, ya sea estudiar, ver la tele, deporte. $Y$ ya te digo que con el deporte se elimina mucho estrés. Fundamentalmente el estrés y la ansiedad es aquí algo normal, teniendo en cuenta que es una situación forzosa, no admitida y que te deja atrapado en una larga espera (comunicación, carta, giro, permiso) y esto hace que tengamos tendencia a pensar en el mañana, (y) no hacemos nada en el presente.

Otros ven en la actividad física y el deporte una práctica que les permite pasar las horas muertas, romper la rutina y superar el aburrimiento que lo invade todo. Así contestaba este preso a la pregunta de por qué hacía ejercicio y deporte:

Porque hay mucho tiempo y no hay mucho más que hacer. Así [con el deporte] te desahogas, comes más y descansas mejor. Además te eleva la autoestima y mejoras tu condición física. Te encuentras más motivado si haces deporte, si no haces nada engordas. Aquí te pones como un cerdo [...] Aquí tienes mucho tiempo para ti, bueno casi todo el tiempo que tienes es para ti y tienes que ocupar el tiempo libre.

Como dice Alex, "uno de los problemas fundamentales de la gente es cómo ocupar el tiempo" que disponen los presos como consecuencia del encierro. Además, resulta especialmente relevante cuando el consumo de droga es uno de los recursos más fáciles para evadirse de la prisión, tal y como repiten muchas internas e internos:

Aquí cuando hago deporte parece que no esté presa y me sirve para desconectar de los problemas. Me siento bien conmigo mismo, no pienso en la heroína. Haciendo deporte pienso lo absurdo que es drogarse. Además, me canso y me siento bien y dejo de pensar en funcionarios e internos y problemas que me joden. (Mujer)

A mí el deporte me hace estar bien físicamente, apartarme de las drogas. El deporte ayuda a dejar las drogas. Además hace que se te vayan los pájaros de la cabeza. Yo en la calle jugaba a futbito y ahora llevo un año y medio haciendo full-contact, y ahora me he apuntado a aikido. Pero en la calle jugaba por diversión. Aquí es diferente. El deporte te equilibra, te hace que dejes la droga. (Hombre) 
Incluso algunos funcionarios de seguridad ven este tipo de beneficios entre los encarcelados:

Para algunos sirven para pasar el tiempo y hacer que la condena se haga más corta. Otros lo que buscan son actividades diarias o salir del módulo. También hay quien hace deporte por ver a quién se encuentra en el polideportivo o, incluso, en los pasillos.

Como señala el funcionario en esta cita, el deporte además de ser percibido como una estrategia contra el tiempo, el aburrimiento y el consumo de drogas permite a los presos escapar del módulo, cambiar de espacio y permitir nuevas relaciones sociales. Para uno de los monitores, se trata "de salir del módulo, de airearse un poco, de relacionarse con la gente" o como señala otro, "la razón principal es salir, ver a la mujer, ver a la querida y ya está. Trapichear [con droga] alguno". Una de las mujeres recluidas lo explicaba de esta manera:

El deporte te ayuda a perder la inactividad, aquí te cansas del mundo, y puedes salir del módulo. Además, conoces a otra gente. Yo en el poli he conocido a gente que me ha dado mucho, que son buenas amistades.

Este tipo de válvula de escape o compensación de los problemas y complejidades diarias se ha utilizado para comprender el papel del deporte en la vida de las personas en libertad (Segrave, 2000). En el contexto de una prisión, sin embargo, la relevancia puede resultar vital, revelándose en una estrategia incluso de supervivencia. De acuerdo con las manifestaciones de los participantes en nuestro estudio, parece que el ejercicio y el deporte contribuyen a paliar los daños del encierro y ayudan a evitar consecuencias más graves. Así lo indican varias investigaciones realizadas en las prisiones de diferentes lugares, ya sea porque mejora la condición física, merma el riesgo de incidentes cardiovasculares o contribuye a reducir el consumo de drogas y de alcohol (Chamarro, Blasco y Palenzuela, 1998; Hitchcock, 1990). Las evidencias en este sentido llevan a Hagan (1989) y Negro (1995) a asegurar que los programas de actividad física mejoran la calidad de vida en la prisión.

De alguna manera, esta cuestión la contempla la normativa legal de las prisiones españolas cuando el Reglamento Penitenciario (1997: 60) señala en su artículo 3.3 que "... la vida en la prisión debe tener como referente la vida en libertad, reduciendo al máximo los efectos nocivos del internamiento...". Igualmente se observa en el Título IX del mismo Reglamento 'De las prestaciones de la Administración Penitenciaria' donde se regulan aspectos relativos a la asistencia sanitaria, a la higiene o a la alimentación. Sin embargo, en ningún lugar se habla de cómo el ejercicio físico y el deporte puede adquirir este significado y pasar a ser una práctica que contribuya a paliar las consecuencias nocivas de la reclusión y mejorar la calidad de vida de los internos de la prisión. 


\section{¿Control social, educación o ambos?}

Cuando el ejercicio y el deporte se realizan por distracción, para llenar el tiempo, aliviar tensiones o evitar problemas, resultan susceptibles de utilizarse como elemento de control social. Así ocurre en la vida cotidiana del ciudadano de a pie y en la vida cotidiana de las personas privadas de libertad. En nuestra etnografía aparece como uno de los significados que emergen, explícita e implícitamente, entre los participantes. En la dimensión más explícita se encuentran los distintos grupos de trabajadores de 'Varoic'. Según Alex, el educador de deportes de la prisión:

Lo ideal sería que el interno, desde que se levanta hasta que se acuesta, esté ocupado en actividades. Por la mañana en actividades deportivas, por la tarde que esté en un taller 0 en actividades culturales. Lo mejor es que esté ocupado y creo que, precisamente, por evitar la ociosidad. Además, es que existe una relación directa entre interno ocioso-interno conflictivo, interno contra la institución. Con internos ocupados desciende totalmente los niveles de conflictividad.

El mismo fenómeno se refleja en las palabras de este funcionario de vigilancia, aunque entre líneas, deseando incluso una mayor extensión de la práctica deportiva entre la población reclusa:

Un porcentaje muy pequeño (practica deporte), cuando convendría que se hiciera mucho, ya que los relaja. El deporte les hace olvidar los problemas, los relaja y los ayuda a evadirse.

La noción de control social surge claramente en las manifestaciones de un monitor que trabaja en la prisión y cuenta lo que le comunican desde la dirección del centro mediante conversaciones informales:

Lo primero que te dicen es que el objetivo no es otro que vayan cansados a la celda y preocupados de todo menos de causar problemas y ya está... les da igual lo que des, como lo des. Les da igual, como si no das nada. "Tú cánsalos, que creen pocos problemas, que por este motivo los sacamos [al polideportivo]". La verdad es que no te dan ninguna norma establecida. Bueno, las normas de no llevar nada de fuera. Pero lo principal es tenerlos entretenidos. En este sentido, estando entretenidos, lo que menos les importa es la actividad que hagas. Por ello, los cursos de actividad física son los más cortos de todos. Eso demuestra el reconocimiento que la actividad física tiene.

El escaso reconocimiento que parece recibir la actividad física y deportiva por parte de la dirección, mencionado de pasada al final de la cita anterior, parece reforzar también el papel de control social asignado. Según la normativa penitenciaria, el deporte es una actividad de Tratamiento más sobre la que descansa la tarea oficialmente educativa de estas actividades. Pero después, en la práctica, reciben el menosprecio y una patente falta de atención. Así se observa en diferentes aspectos del funcionamiento diario de la 
prisión como la falta de educadores de deporte, el poco caso que hacen a la planificación y desarrollo de las actividades físicas y deportivas y la ausencia del único educador de deportes en la Junta de Tratamiento ${ }^{2}$. Los responsables del área están más preocupados por el número de actividades y participantes que por su orientación y calidad. Tal y como el educador de deportes relata:

No me exigen: debe haber un nivel de internos en actividades deportivas, debemos conseguir tal... No me exigen ni me piden ningún objetivo. Los objetivos se hacen o no se hacen, pero nadie me dice que debo conseguir algo. No sé si es que confían mucho en mí o 'pasan'.

Los presos también son conscientes de que el deporte juega un papel de control social a los ojos de los empleados de la institución, pero valoran la ayuda que les proporciona como queda reflejado en esta cita:

[...] creo que principalmente se usa como válvula de escape por parte de la Administración. No se usa como potenciación de la persona, debería estar más valorado [...] El deporte palia los daños del encierro. Tanto los físicos como los psicológicos. Porque los psicológicos no se ven pero están ahí y los responsables saben que el deporte libera a la gente. Son conscientes de ello y usan ese recurso para evitar problemas. Aunque también es en beneficio tuyo.

Otros ven, entre los beneficios del deporte, un cierto potencial educativo proporcionado por el ethos deportivo del autocontrol y la formación del carácter. Como decía un preso, "el deporte, si te llena de ilusión, si lo coges con ganas, te ayuda. A mí me ha quitado el odio que llevaba dentro, me ha dado autocontrol". El subdirector de otra prisión española que también fue entrevistado lo expresaba de esta manera:

Una persona que es capaz de llevar una actividad reglada, donde suelta adrenalina, y que la controla con sus normas, con las normas del deporte en cuestión, el que sea, pues de alguna forma es capaz de autocontrolarse que es, en definitiva, lo que se pretende con la educación. Si es capaz de autocontrolarse es capaz de dominar una situación externa.

La verdadera contribución educativa del deporte en la prisión la señalan cuando el autocontrol trasciende la práctica deportiva y llega a la vida cotidiana, tal y como apunta Alex, el educador de deportes:

${ }^{2}$ Según el artículo 272 del Reglamento Penitenciario (1997), las Juntas de Tratamiento deben estar integradas por el director, el subdirector de tratamiento, el subdirector médico, el director de la Unidad Docente, el coordinador de los Servicios Sociales y los técnicos, educadores y jefes de servicios que hayan intervenido en las propuestas a debatir. En nuestro caso, y como reconoce el subdirector de tratamiento, "a lo mejor por la celeridad con que se dan [las Juntas de Tratamiento] no se trata la actividad física con el detenimiento que debiera". El propio Alex dice; "a mí nunca me llama la Junta ni estoy en reuniones para informar de aspectos deportivos". 
Cuando mayor es el [auto]control mayor es el potencial de modificación de conducta [...] El full-contact es un estilo de vida que trasciende las horas que tú le dedicas, el arte marcial es un estilo de vida. Trasciende las horas de entrenamiento, debes coger un hábito, es un estilo de vida que te permite mejorar.

Este énfasis educativo lo encontramos especialmente acusado entre los practicantes de las artes marciales que, con Alex a la cabeza, forman parte de un club federado de full-contact. Las citas de estos dos presos mencionan la influencia del arte marcial en la formación de un carácter positivo que les permite canalizar la agresividad:

[...] las artes marciales te hacen el carácter más serio. No es como el fútbol o el baloncesto que puedes estar diciendo tonterías o gritando en el campo. Requiere concentración, seriedad. La gente, al principio, se puede 'rebotar' pero con el tiempo van cogiendo el espíritu marcial y se van haciendo serios.

Las artes marciales canalizan la agresividad, hacen de la agresividad algo lúdico, se le da una finalidad lúdica. Así se controla y te da seguridad. Se aprende a ser agresivo unas horas a la semana. Sin embargo, antes y después, hay una sonrisa. Es una finalidad deportiva, es muy positivo.

Hay que puntualizar que las artes marciales son rechazadas por algunos trabajadores de la prisión, funcionarios de vigilancia y monitores principalmente, porque las ven potencialmente muy peligrosas. Sus argumentos se refieren al posible uso que los presos puedan hacer de estas técnicas en un futuro, tanto dentro como fuera de la prisión, mientras que no lo ven en otras prácticas que mejoran la fuerza como es el caso del culturismo. Estas citas sirven de ejemplo:

[el full-contact] Es un deporte que enseña a agredir. Si sabe de dónde cogerte, si está preparado te puede causar muchos problemas (funcionario de vigilancia).

[...] los deportes de contacto que los quiten cuanto antes mejor porque son peligrosísimos

[...] Un día hablando me decían que no, que no lo iban a usar. Y quién te dice a ti que cuando salgan no se van a liar a patadas (monitor).

Probablemente por eso los interesados en las artes marciales son los más fervientes defensores del potencial educativo y de autocontrol de las prácticas deportivas. Sin embargo, inevitablemente, sobre las manifestaciones anteriores planea de nuevo el papel de control social que juegan las actividades físicas y deportivas dentro de la prisión. En última instancia, como ya señalaba Grayzel (1978), las actividades en la prisión y sus participantes asumen la dinámica de control a través de los castigos y las recompensas. En este sentido, el deporte y el ejercicio físico se ven atrapados y mediatizados por el área de seguridad de la prisión. Así lo relata un monitor y una monitora que trabajan en 'Varoic':

Aquí van pues, por ejemplo, a los cursillos porque les dan un vis-a-vis extra. Entonces, saben que si acaban el curso les dan el vis-a-vis. Es que si tienes módulos conflictivos, 
como el 10, donde te juegas la vida casi todos los días y les dices 'si te llevas bien a fullcontact', pues es que la mayoría del 10 están en full (monitor).

Si te comportas bien vas al poli y si te portas aún mejor te hacen monitor. Pero eso pasa en el poli y también en las otras actividades, en el teatro pasa igual. Si tienes un buen comportamiento, si te llevas bien con la monitora de teatro y tal, lo mismo. Y en la escuela igual, en el graduado. Creo que en general funciona todo así. Desde mi punto de vista creo que no es un buen procedimiento pero es verdad que las cosas se hacen de esta manera (monitora).

La implicación de presos y presas, inconsciente o no, en el sistema de control de la prisión se observa de forma evidente en la selección de los y las participantes en las actividades deportivas. En este sentido, tener un buen comportamiento es el principal criterio para ser seleccionado, de forma que llevarse bien obtiene una buena recompensa (no olvidemos que tan sólo un 10\% aproximadamente accede a los deportes del Tratamiento). Este fenómeno es muy evidente en las artes marciales donde, aparte de criterios estrictamente físicos, se exige "un expediente de conducta intachable" y, además, "la naturaleza del delito". Es decir, "que no estén condenados por delitos de carácter violento, lesiones, homicidios y delitos contra las persona en general". Los 'destinos' o aquellos presos que colaboran de varias formas en el funcionamiento del polideportivo también participan en la selección de los participantes. Para Alex resulta clave el conocimiento y "las confidencias que te pueden dar los internos destinados en el polideportivo" sobre los aspirantes a entrar en el club de artes marciales y en otras actividades deportivas. En definitiva, con este tipo de participación los 'destinos' no sólo forman parte de la dinámica de castigos y recompensas que atañen a los aspirantes, sino también de la manera en que ellos mismos se ganan el puesto, viéndose arrastrados por el bucle del control social imperante. En cierta forma, evidencia un alto grado de 'prisionización' entre dichos internos, es decir, el nivel de asimilación de las normas sociales que permite vivir a los presos y presas entre rejas (Muñoz, 1990; Ríos, 2004).

\section{Comentarios finales}

Desde que los sistemas penitenciarios de los países democráticos incluyeron la reeducación de las personas presas y la facilitación del retorno exitoso a la sociedad como meta final del internamiento, el debate sobre sus posibilidades y forma de llevarlo a cabo no ha dejado de arreciar. Si bien los textos legales la recogen como finalidad oficial de los sistemas penitenciarios, otros consideran sencillamente que dicha meta no ha dejado de ser utópica (Matthews, 2003). Este debate y unas posiciones equivalentes se reproducen al abordar el papel que puede jugar el ejercicio físico y el deporte en las prisiones. Para algunos autores, los programas recreativos, incluidos los deportivos, pueden reducir el crimen y son esenciales para la rehabilitación social de los presos (O’Morrow y Reynolds, 1989). En cambio otros, no sólo se muestran en desacuerdo con 
esta idea, sino que consideran que las prácticas físicas y deportivas pueden aumentar la agresividad y el comportamiento antisocial (Drinkwater, 1980).

Después de escuchar la voz de los participantes de nuestro estudio, la reinserción aparece como una quimera o una empresa titánica para héroes individuales. Aún así, los presos y presas otorgan importantes beneficios a la práctica física y deportiva, aunque no consideran que evite la reincidencia delictiva. Parece bastante ambicioso conceder esta posibilidad al ejercicio y el deporte, al menos en exclusiva, cuando sólo se contribuye a ello con algún curso de monitor o socorrista que pudiera serles útil más allá de la prisión. En este sentido, nuestro estudio no comparte el optimismo de otros trabajos que mencionan la reinserción social como un objetivo alcanzable mediante las prácticas deportivas (Castillo, 2005 y 2006; Chamarro, 1993), aunque también se mencionan otros beneficios para sobrellevar la vida carcelaria, similares a los señalados en dichas investigaciones. Es decir, aunque el deporte contribuya a dejar el consumo de drogas entre las personas presas, no podemos concluir que contribuya a la reinserción social mientras no se realice un seguimiento posterior al internamiento y dichas personas se (re)integren en la vida social.

Probablemente, un nivel de influencia intermedio correspondería al potencial (re)educativo del deporte ligado al autocontrol que los participantes en nuestro estudio asocian fundamentalmente al respeto de las reglas. Como señalara Cagigal (1990: 85), "es el primer gran principio de la convivencia humana" porque de lo contrario "le será impuesto desde fuera". Se trata, por tanto, de una influencia más sutil que la reinserción porque, según sus defensores, el autocontrol ligado al cumplimiento de las reglas cala individualmente hasta formar parte del carácter y la vida cotidiana de los reclusos. Así se manifiestan algunos cargos directivos y los destinos deportistas de artes marciales, tal y como han hecho muchas otras personas desde la aparición del deporte moderno en el siglo XIX. No obstante, ya hemos visto que algunos guardias de seguridad y monitores no confían tanto en ese autocontrol aprendido, al menos en relación con las artes marciales, debido al peligroso uso que puedan hacer de sus aprendizajes dentro y fuera de la cárcel. Aun así, están entre las creencias socialmente arraigadas las de que el deporte infunde una serie de valores morales deseables para la formación del carácter de las personas y que el aprendizaje del deporte sirve de preparación para la vida social.

Sin embargo, se trata de ideas muy criticadas porque las reglas que gobiernan los deportes son reglas funcionales y no reglas morales (Bailey, 1975) y el apoyo empírico parece ir en sentido opuesto a la transmisión de valores deseables (Greendorfer, 1987; Coakley, 2004). Además, existen claras diferencias entre el deporte y la vida cotidiana. Aunque en ambos casos la gente se enfrenta a retos, se trata de retos diferentes. En la vida cotidiana, raras veces nos enfrentamos directamente a nuestros oponentes ni sabemos cuándo se ha eliminado el reto o si hemos finalmente conseguido la victoria. En cambio, en el deporte, los oponentes se enfrentan directamente, no hay dudas sobre el momento en que finaliza el juego y quién ha conseguido la victoria. La vida de todos los días es compleja, muy ambigua e incierta y difícil de comprender, mientras que el deporte es simple, poco 
ambiguo e incierto y fácil de comprender (Valentine, 1980). Asimismo, las acciones de la vida cotidiana poseen un componente moral real y sus consecuencias son potencialmente muy serias, mientras que las acciones en el deporte tienen un componente moral que normalmente se circunscribe a una situación deportiva muy particular y sus consecuencias no van más allá del ámbito deportivo. Sólo en el caso de los deportistas profesionales puede haber cierta superposición por tratarse de su medio de vida.

El deporte, como cualquier otra actividad de la vida, podrá transmitir valores deseables o no deseables y trasladarse a la vida cotidiana de los implicados, dependiendo del tipo de relaciones interpersonales del contexto social de su práctica (Devís, 1995; Gutiérrez, 2003) que, en nuestro caso, es la vida carcelaria. No será, por tanto, una transmisión automática derivada de la simple práctica deportiva sino que dependerá de lo que ocurra alrededor de la misma (Shields y Bredemeier, 1995). Sólo con la existencia de un proyecto, con propósitos, contenidos y estrategias metodológicas, pensado y dirigido explícitamente a la promoción de valores morales será posible la existencia de relaciones sociales positivas como para poder hablar de (re)educación. En nuestro estudio, en concreto, no se observa un proyecto de estas características en las actividades deportivas del Tratamiento, a pesar de que el educador de deportes reclame insistentemente más atención y consideración de las autoridades para sus prácticas. Más bien nuestros sujetos asignan valores al deporte que se transmite a los deportistas por el simple hecho de participar. En cambio, cuando existen programas especialmente diseñados para influir en la reeducación, los resultados parecen ser más positivos, tal y como se señalan en otros estudios (Castillo, 2005 y 2006; Chamarro, 1993).

Donde existe poca discusión, tanto en la literatura como en las manifestaciones de nuestros actores, es en el papel de entretenimiento y compensación que juega el deporte y el ejercicio físico. Pero en nuestro caso, en un sentido que va más allá del papel que recibe en condiciones de libertad. En un contexto penitenciario, el entretenimiento y la compensación surgen, particularmente, para superar la experiencia cotidiana del encierro obligatorio, probablemente la dimensión más física y corporal del castigo que supone la reclusión. Las prácticas físicas y deportivas se convierten así en una especie de 'evasión' simbólica de la cárcel, en concreto, evasión de la experiencia cotidiana del espacio y el tiempo mientras dura la condena. Esto resulta evidente para quienes sufren el encierro y también para quienes trabajan en la prisión. Recordemos que la división de la prisión en módulos y estancias separadas limita enormemente la libertad de movimientos de los presos y las actividades deportivas ofrecen posibilidades espontáneas de movimiento y relación social en dos de los espacios de mayor libertad y dominio personal de la prisión, el patio y el polideportivo. El tiempo representa la otra experiencia cotidiana vital de la reclusión, esencia misma de la pena para los internos, ya que las condenas no son, ni más ni menos, que determinadas cantidades de tiempo (Matthews, 2003). En la prisión, el tiempo se gana y se pierde, se comercia con él y la prioridad es que pase lo más rápidamente. Como el deporte también transcurre en un tiempo determinado, la participación en las prácticas físicas del Tratamiento se convierte en un auténtico 
privilegio porque es una de las pocas oportunidades que tienen los presos para ocuparlo y huir del hastío de la cárcel.

Sin embargo, en manos de la autoridad penitenciaria, el espacio y el tiempo parecen ponerse de acuerdo para evitar problemas y la manera en que se encuentran estructurados en las prisiones obedece a unas claras intenciones de orden y control. Por lo tanto, las prácticas físicas y deportivas, así como el polideportivo, se convierten en pequeños espacios de libertad con los que se negocia, mediante el bucle de castigos y recompensas que apuntaba Grayzel (1978), para mantener ocupados (y controlados) a los reclusos. Si el deporte puede servir, a nivel individual, de liberación para las personas encarceladas, se torna en control social desde la perspectiva de la autoridad penitenciaria. La escasa atención que recibe el deporte como actividad educativa, es decir, como actividad intencional con objetivos y estrategias metodológicas, contribuye a reforzar la idea de que su papel consiste en mantener ocupados a los presos. No es de extrañar que el educador de deportes se manifieste desesperanzado por la indiferencia de su labor en la cárcel (falta de personal y desprecio por objetivos y logros). Pero cuando los presos y presas valoran esta actividad, inmediatamente se convierte en un objeto preciado, excusa para mostrar un buen comportamiento y recompensa de la institución en su estrategia de control social. Es más, esta cuestión se extiende al conjunto de actividades y programas del Tratamiento que reciben mayor reconocimiento que el ejercicio físico y el deporte. En este caso se debe al dominio que ejerce el Régimen disciplinario sobre todos los aspectos de la vida en 'Varoic', tal y como lo ha vivido el investigador principal de esta etnografía, y reflejan además de lo expuesto hasta ahora, las siguientes observaciones:

- Las actividades que se llevan a cabo en el polideportivo se suspenden de forma inmediata cuando falta el funcionario que vigila desde la garita de entrada al recinto

- El cumplimiento de los horarios del polideportivo es estricto, no pudiéndose alterar bajo ningún concepto, aún cuando se estén celebrando acontecimientos deportivos.

- La colaboración de los funcionarios de seguridad en el Tratamiento, que por otra parte manda la ley, es nulo, limitándose éstos a abrir y cerrar las puertas y poco más.

- El subdirector del área de tratamiento no forma parte de la Comisión Disciplinaria de la prisión, aunque la imposición de sanciones afecte al tratamiento de presos y presas.

En cierta forma, el Régimen le gana la partida al Tratamiento, ya que el orden dentro de la cárcel, representado sobre todo por la evitación de conflictos y fugas, es la razón de ser de todo lo que se organiza. Cuando una actividad educativa conlleva algún riesgo y pone en entredicho la organización regimental, sencillamente se elimina. Por eso, en cierta manera, el ejercicio físico y el deporte viven atenazados por la realidad del contexto donde se imparte y legitiman inevitablemente el estado de las cosas, aunque a nivel individual pueden jugar un papel de 'evasión' simbólica y liberación personal. 


\section{REFERENCIAS BibLIOGRÁFICAS}

Alarcón, J. (1987), "Tratamiento penitenciario", en V. Garrido y M. Vidal (coords.), Lecturas de pedagogía correccional, Valencia, Nau llibres, pp. 201-214.

Bailey, C. (1975), "Games, winning and education", Cambridge Journal of Education, 5:40-50.

Bolívar, A., J. Domingo y M. Fernández (2001), La investigación biográfico-narrativa en educación. Enfoque y metodología, Madrid, La Muralla.

Cabrera, P. J. y J.C. Ríos (1998), Mil voces presas, Madrid, Universidad Pontificia de Comillas.

Cagigal, J.M. (1990), Deporte y agresión, (1. a Edición de 1976) Madrid, Alianza Editorial y Consejo Superior de Deportes.

Caplan, A. (1996), The role of recreational sports in the Federal Prison System, Wolfville (Canadá), Acadia University, Master of Arts and Sociology Thesis.

Castillo, J. (2005), Deporte y reinserción penitenciaria, Madrid, Consejo Superior de Deportes, Centro de Alto Rendimiento y de Investigación en Ciencias del Deporte.

(2006), "Valores sociales del deporte en el ámbito penitenciario", en Pujadas, X., A. Fraile, V. Gambau, F. X. Medina y J. Bantulà (comps.), Culturas deportivas y valores sociales (VIII Congreso AEISAD), Madrid, Lib. Deportivas Esteban Sanz, pp. 121-129.

Chamarro, A. (1993), "Deporte y ocio para la reinserción de reclusos: La experiencia del Centro Penitenciario de Pamplona", Revista de Psicología del Deporte 4: 87-97.

Chamarro, A., T. Blasco y D. Palenzuela (1998), "La práctica de ejercicio en las prisiones: factores asociados a la iniciación y el mantenimiento", Revista de Psicología del Deporte 13: 69-91.

Coakley, J. J. (2004), Sport in society. Issues and controversies, 8a Edición, Boston, McGraw Hill.

Coffey, A. y P. Atkinson (1996), Making Sense of Qualitative Data, Thousand Oaks Sage Publications.

Constitución Española (1983), Constitución Española de 1978, Valencia, Generalitat Valenciana.

Courtenay, W.H. y D. Sabo (2001), "Preventive health strategies for men in prison", en Sabo, D., T. A. Kupers y W. London, Prison Masculinities, Philadelphia, Temple University Press, pp. 157-172.

Devís, J. (1995), "Deporte, educación y sociedad: hacia un deporte escolar diferente", Revista de Educación 306: 455-472.

Drinkwater, J.B. (1980), "The development and manifestation of aggression with special references to competitive sport", Recreation Research Review 8: 2: 41-44.

Fetterman, D. (1998), Ethnography. Step by step, Londres, Sage Publications.

Flick, U. (2004), Introducción a la investigación cualitativa, A Coruña, Fundación Paideiai Galiza. 
Foucault, M. (1978), Vigilar y Castigar. Nacimiento de la prisión, Madrid, Siglo Veintiuno Editores.

Goodson, I. y C. Fliesser (1995), "Negotiating fair trade: Towards collaborative relationships between researchers and teachers in College dettings", Peabody Journal of Education 70: 5-17.

Grayzel, J. (1978), "The Functions of Play and the Play Motif at a State Penitenciary", en Salter, M. (ed.), Play Anthropological Perspectives, Nueva York, Leissure Press, pp. 94-103.

Greendorfer, S. (1987), "Psycho-social correlates of organized physical activity", Journal of Physical Education, Recreation and Dance, 58: 59-64.

Gutuérrez, M. (2003), Manual sobre los valores en la educación física y el deporte, Barcelona, Paidós.

Hagan, J. (1989), "Role and significance of sport/recreation in the penal system", Prison Service Journal 75: 9-11.

Hammersley, M. y P. Atkinson (1994), Etnografía. Métodos de investigación, Barcelona, Paidós.

Hitchcok, H. (1990), "Prisons. Exercise versus recreation", Journal of Physical Education, Recreation and Dance 61, 6: 84-88.

L.O.G.P. (1979), "Ley Orgánica 1/1979 de septiembre, General Penitenciaria”, en Bases de datos AranzadiLegislación 30/86, Pamplona, Aranzadi (soporte informático).

Manzanos, C. (1992), Cárcel y marginación social, Donostia, Gakoa.

Matthews, R. (2003), Pagando tiempo. Una introducción a la sociología del encarcelamiento, Barcelona, Edicions Bellaterra.

Maykut, P.y R. Morehouse (2000), Beginning Qualitative Research. A philosophic and practical guide, London, Routledge/Falmer.

Mejía, R. (2002), "El microanálisis en el estudio de la mediación sociocultural de procesos cognoscitivos", en Mejía, R. y S.A. Sandoval (coords.), Tras las vetas de la investigación cualitativa, Guadalajara, Iteso, pp. 101-122.

Mortimer, G. (1999), "Mind games", Rugby World 78: 78-81.

Muñoz, F. (1990), "Tratamiento penitenciario: Utopía no alcanzada o simple quimera", En VI Jornadas penitenciarias andaluzas, Sevilla, Consejería de Gobernación, pp. 37-43.

Negro, C. (1995), "Actividades en los centros penitenciarios", en RODRÍGUEZ, P. y J. A. Moreno (dirs.), Perspectivas de actuación en Educación Física, Murcia, Universidad de Murcia, pp. 229-245.

O'Morrow, G. S. y R. P. Reynolds (1989), Therapeutic Recreation: A Helping Profession, Englewood Cliffs, Prentice-Hall Inc.

Reglamento Penitenciario (1997), "Reglamento Penitenciario", en Ley y Reglamento Penitenciario, Madrid, Civitas, pp. 56-204. 
Rhodes, L. (2001), "Toward an anthropology of prisons", Annual Review of Anthropology 30: 65-83.

Ríos, M. (2004), "La educación física en los establecimientos penitenciarios de Catalunya”, Tàndem. Didáctica de la Educación Física 15: 69-82.

Sabo, D. (2001), "Doing time, doing masculinity: sports and prison", en Sabo, D., T. A. Kupers y W. London, Prison Masculinities, Philadelphia, Temple University Press, pp. 61-66.

Segrave, J. (2000), "Sport as escape", Journal of Sport and Social Issues 24, pp. 61-77.

Shields, D. L. y M. J. Bredemeier (1995), Character development and physical activity, Champaign, Human Kinetics.

Sparkes, A.C. (2002), Telling tales in sport and physical activity. A qualitative journey, Champaign, Human Kinetics.

Taylor, S. J. y R. Bogdan (1986), Introducción a los métodos cualitativos de investigación. La búsqueda de significados, Barcelona, Paidós.

Valentine, J. (1980), "Sport and ideology: some distinctions and considerations", ARENA Review 4: 7-12.

Valverde, J. (1991), La cárcel y sus consecuencias, Madrid, Ed. Popular.

Wagner, M., R. McBride y S. Crouse (1999), "The effects of weight-training exercise on aggress", Prison Service Journal 79, pp. 72-89.

Wolcott, H. (1973), The man in the principal's office. An Ethnography, Eugene, University of Oregon.

(1990), Writing up qualitative research, Newbury Park, Sage Publications.

Woods, P. (1998), La escuela por dentro: la etnografia en la investigación educativa, Barcelona, Paidós.

RECIBIDO: $18 / 02 / 07$

ACEPTADO: $13 / 12 / 07$ 